\title{
Radio signatures of shock-accelerated electron beams in the solar corona
}

\author{
G. Mann ${ }^{1}$, V. N. Melnik ${ }^{2}$, H. O. Rucker ${ }^{3}$, A. A. Konovalenko², and A. I. Brazhenko ${ }^{4}$ \\ 1 Leibniz-Institut für Astrophysik Potsdam (AIP), An der Sternwarte 16, 14482 Potsdam, Germany \\ e-mail: GMann@aip.de \\ 2 National Academy of Sciences of Ukraine, Institute of Radio Astronomy of NAS, 4 Chervonopraporna Str., 61002 Kharkov, \\ Ukraine \\ 3 Commission for Astronomy, Austrian Academy of Sciences, Schmiedlstrasse 6, 8042 Graz, Austria \\ 4 Poltava Gravimetrical Observatory of Institute of Geophysics of NASU, Myasoedova, 27/29, 36014 Poltava, Ukraine
}

Received 2 February 2017 / Accepted 11 November 2017

\begin{abstract}
Context. The Sun's activity can appear in terms of radio bursts. In the frequency range $8-33 \mathrm{MHz}$ the radio telescope URAN-2 observed special fine structures appearing as a chain of stripes of enhanced radio emission in the dynamic radio spectrum. The chain drifts slowly from 26 to $23 \mathrm{MHz}$ within $4 \mathrm{~min}$. The individual structures consist of a "head" at the high-frequency edge and a "tail" rapidly drifting from the "head" to lower frequencies over an extent of $\approx 10 \mathrm{MHz}$ within $8 \mathrm{~s}$. Since they resemble the well-known "herring bones" in type II radio bursts, they are interpreted as shock accelerated electron beams.

Aims. The electron beams generating these fine structures are considered to be produced by shock drift acceleration (SDA). The beam electrons excite Langmuir waves which are converted into radio waves by nonlinear wave-plasma processes. That is called plasma emission. The aim of this paper is to link the radio spectral data of these fine structures to the theoretical results in order to gain a better understanding of the generation of energetic electrons by shocks in the solar corona.

Methods. Adopting SDA for generating energetic electrons, the accelerated electrons establish a beam-like velocity distribution. Plasma emission requires the excitation of Langmuir waves, which is efficient if the velocity of the beam electrons exceeds a few times thermal electron speed. That is the case if the angle between the shock normal and the upstream magnetic field is nearly perpendicular. Hence, the Rankine-Hugoniot relationships, which describe the shock transition in the framework of magnetohydrodynamics, are evaluated for the special case of nearly perpendicular shocks under coronal circumstances.

Results. The radio data deduced from the dynamic radio spectrum can be related in the best way to the theoretical results, if the electron beams, which generate these fine structures, are generated via SDA at an almost perpendicular shock, which is traveling nearly horizontally to the surface of the Sun.
\end{abstract}

Key words. Sun: flares - Sun: radio radiation - Sun: particle emission - shock waves - acceleration of particles - plasmas

\section{Introduction}

The Sun's activity appears not only in the well-known 11yr sunspot cycle but also in eruptive events such as flares and coronal mass ejections, as well as radio bursts. All these events are accompanied by an enhanced radio emission. Therefore the study of the Sun's radio radiation provides important information on plasma processes associated with the Sun's activity.

On April 7, 2011, special fine structures (see Fig. 1) were recorded in the solar radio radiation in the frequency range 8-33 MHz with the radio spectrometer at the radio telescope URAN-2 of Poltava Gravimetrical Observatory of Institute of Geophysics of National Academy of Sciences of Ukraine (Brazhenko et al. 2005). Figure 1 shows the dynamic radio spectrum of these fine structures (Brazhenko et al. 2012). They appear as a chain of stripes of enhanced radio emission with a pronounced circular polarization. The chain drifts slowly from high towards low frequencies. The individual structures consist of two components namely the "head" at the high-frequency edge and the "tail" extending to lower frequencies (see also Fig. 2). In other events in the decameter range, sometimes opposite cases are observed, that is, the heads appear at the lowfrequency edge, whereas the tails drift rapidly towards higher frequencies (V. N. Melnik, priv. comm.). In the case discussed here, the high frequency edges of these heads drift slowly from $26 \mathrm{MHz}$ towards $23 \mathrm{MHz}$ in the period 11:44-11:48 UT (see Fig. 1) leading to a drift rate $D_{\mathrm{f}, \mathrm{sd}}=-12.5 \mathrm{kHz} / \mathrm{s}$. The tails extend over a frequency range of $\approx 10 \mathrm{MHz}$ with drift rates of about $D_{\mathrm{f}, \text { tail }}=-1.25 \mathrm{MHz} / \mathrm{s}$ (see Fig. 2).

These structures resemble the "herring bones" that appear as fine structures in solar type-II radio bursts. In dynamic radio spectra, solar type-II radio bursts manifest themselves as stripes of enhanced radio emission slowly drifting from high to low frequencies and are considered as the radio signature of shock waves traveling through the corona (Smerd et al. 1962; Wild \& Smerd 1972; see as reviews e.g. Nelson \& Melrose 1985; and Mann 1995). Approximately, 21\% of all type-II radio bursts show these so-called herring bones (Cane \& White 1989). They are rapidly drifting emission stripes shooting away from the slowly drifting "backbone" towards lower and higher frequencies (see e.g., Nelson \& Melrose 1985; Cairns \& Robinson 1987). Herring bones are regarded as the radio signature of electron beams generated by the shock wave associated with the backbone (see e.g., Nelson \& Melrose 1985; Mann 1995).

The fine structures shown in Fig. 1 can be considered as individual herring bones due to the following reasons. 


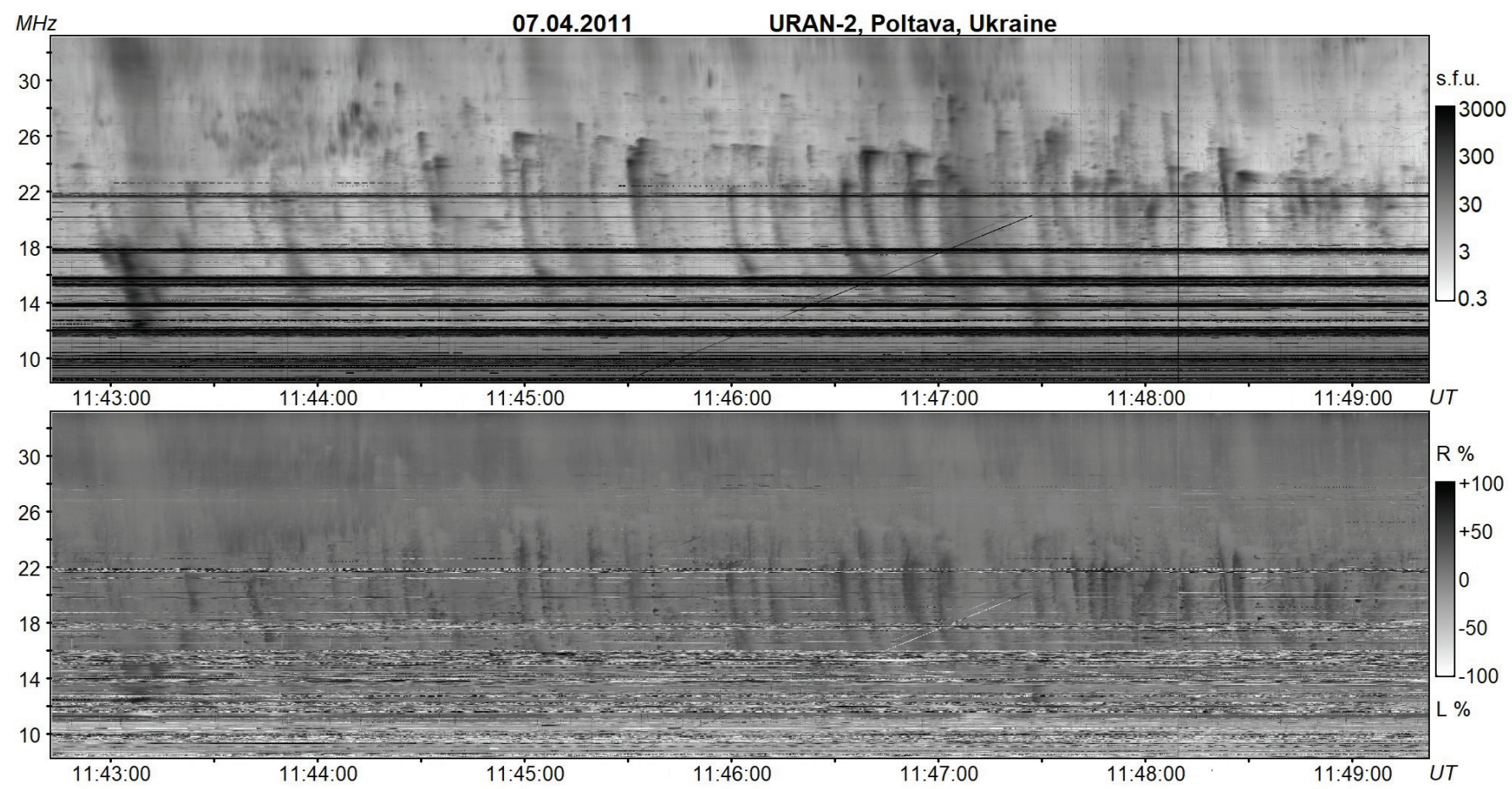

Fig. 1. Dynamic radio spectrum in the frequency range $8-33 \mathrm{MHz}$ as recorded during the period 11:43-11:50 UT on 7 April 2011 with the gray-scaled intensity (top), which is given in solar flux units ( 1 s.f.u. $=10^{-22} \mathrm{~W} \mathrm{~m}^{-2} \mathrm{~Hz}^{-1}$ ), and degree of circular polarization (bottom).

- Cairns \& Robinson (1987) described herring bones (HB) in dynamic radio spectra: “... while the duration of a typeIII burst invariably increases towards lower frequencies, resulting in a fan-like shape, a HB burst has a longer duration nearer the starting frequency, thereby resembling a thorn. This observation allows us to safely distinguish normal HB bursts from the type-III-like bursts ..." (see Cairns \& Robinson 1987, p. 367). The fine structures discussed here show the same spectral morphology, that is, a thorn-like shape (or in other words a head-tail structure).

- HBs exhibit a pronounced circular polarization (Stewart \& Magun 1980; Suzuki et al. 1982; Cairns \& Robinson 1987; Dorovskyy et al. 2015). The fundamental radiation of HBs is usually more circularly polarized than the harmonic one (Cairns \& Robinson 1987). The fine structures discussed here show also a significantly circular polarization as seen at the bottom of Fig. 1 .

- The slow drift of $-12.5 \mathrm{kHz} / \mathrm{s}$ is typical for type-II bursts in the decameter range (see e.g., Mann 1995; Melnik et al. 2004; Dorovskyy et al. 2015).

- The fast drift rate of the tails of $-1.25 \mathrm{MHz} / \mathrm{s}$ is typical of HBs in the decameter range (Melnik et al. 2004; Dorovskyy et al. 2015). We note that the drift rate of type-III bursts is roughly about $-2.36 \mathrm{MHz} / \mathrm{s}$ at $20 \mathrm{MHz}$ according to Alvarez \& Haddock (1973). Typically, HBs have a lower drift rate than type-III bursts in the same frequency range (Mann \& Klassen 2002).

Since the fine structures discussed here show all typical features of HBs in the decameter range, it is justified to also consider them as the radio signature of shock-accelerated electron beams (Nelson \& Melrose 1985; Cairns \& Robinson 1987; Zlobec et al. 1993; Mann 1995, 2006; Aurass 1996; Cairns et al. 2003; Nindos et al. 2008; Dorovsky et al. 2015). Hence, the slow drift of the high-frequency edge of the chain of heads and the rapid drift

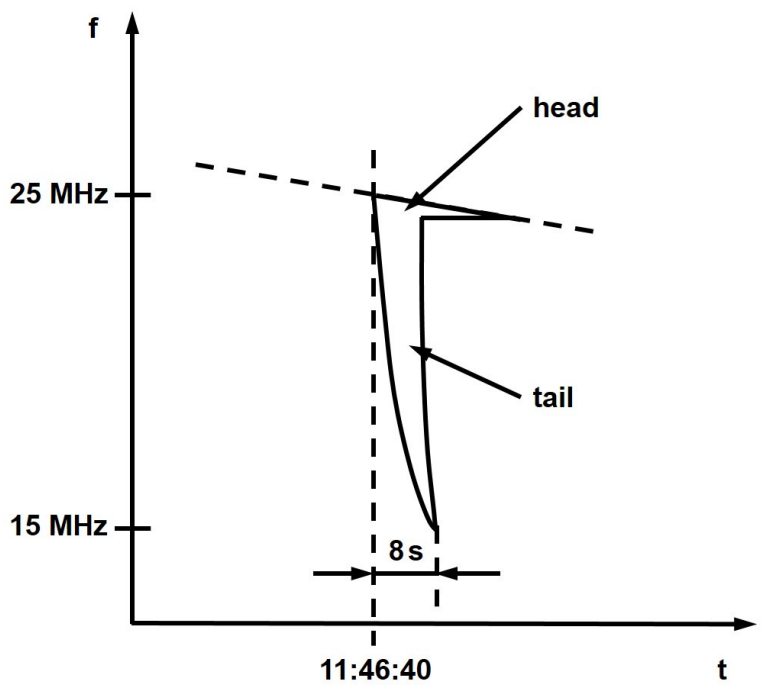

Fig. 2. Sketch of the shape of the fine structures discussed here.

of the tails represent the radio signature of the associated shock wave propagating outwards in the corona and the shock accelerated electron beams shooting away from the shock, respectively.

The structures of discussion are superimposed on a longlasting type-IV continuum accompanied by a behind-limb coronal mass ejection (CME). It is not unusual for type-II bursts to occur with a type-IV continuum as reported by Melnik et al. (2008) and Dorovskyy et al. (2015).

In the solar corona, shock waves can be generated either by the blast wave due to the pressure pulse of a flare (Uchida et al. 1973; Vršnak et al. 1995; Magdalenic et al. 2012) or driven by a CME (Stewart et al. 1974a, 1974b; Melnik et al. 2008; Dorovskyy et al. 2015); they can be observed as type-II 
radio bursts in the metric and decameter wave ranges (Wild \& McCready 1950; Uchida 1960; Melnik et al. 2004; Dorovskyy et al. 2015).

The mechanism by which electrons are accelerated up to high energies is currently under debate, since energetic electrons are the source of the nonthermal radio and X-ray radiation. The aim of this paper is to examine what can be learnt from these radio observations for the generation mechanism of energetic electrons at shocks in space. Here, we assume that the electrons are accelerated by shock drift acceleration (SDA; see e.g. Holman \& Pesses 1983; Ball \& Melrose 2001; Mann \& Klassen 2005).

The radio telescope URAN-2 is briefly described in Sect. 2, where the observational data deduced from the dynamic radio spectrum in Fig. 1 are also presented. Data analysis is presented in Sect. 3. The necessary conditions of radio emission via plasma emission under coronal circumstances are discussed in Sect. 4. In Sect. 5, the SDA is briefly introduced and the properties of shock accelerated electron beams are derived. The link between the observational data and the theoretical results is discussed in Sect. 6. A Summary is presented in Sect. 7. A summary of the Rankine-Hugoniot relationships describing the relation between the physical quantities in the up- and downstream regions of a shock is given in Appendix A.

\section{Observations}

The observations were performed with the radio telescope URAN-2 (Brazhenko et al. 2005) of the Poltava Gravimetrical Observatory of the Geophysics Institute in the National Academy of Sciences of Ukraine. The telescope was built near Poltava (Ukraine) in 1992 and is part of the all-Ukrainian interferometer URAN (Ukrainian Radio Interferometer of National Academy of Sciences). The radio telescope consists of 512 broadband dipoles. The telescope area comprises $238 \mathrm{~m} \times 118 \mathrm{~m}$ (corresponding to $28000 \mathrm{~m}^{2}$ ). The beam of the radio telescope is $7^{\circ} \times 3^{\circ}$ at $25 \mathrm{MHz}$ and is operated electronically. The working frequency band is from 8 to $33 \mathrm{MHz}$. The radio telescope URAN-2 has the capacity to measure polarization of radio emission. It is used both as a part of the interferometer for observations of extragalactic objects such as quasars and radio galaxies, and as an autonomous instrument for observations of the Sun and Jupiter. Observations of solar radio emission are carried out daily at intervals of approximately $3 \mathrm{~h}$ and more centered to the meridian. The tracking of the Sun is automatic with a switch frequency of once every four minutes. DSP (Digital Spectral Polarimeter; Ryabov et al. 2010) with time resolution 1-100 ms and frequency resolution $4 \mathrm{kHz}$ was used in solar observations during 2011. The dynamic range of DSP is $90 \mathrm{~dB}$. The sensitivity of the radio telescope URAN-2 is about 500 Jy during solar observations.

With this instrument, special fine structures are observed on April 7, 2011, as presented in Fig. 1. In dynamic spectra, the morphology of such individual structures is illustrated in Fig. 2. These consist of a "head" at its high frequency edge and a "tail" rapidly drifting towards lower frequencies. The high frequency edges (heads) of these structures drift from $26 \mathrm{MHz}$ to $23 \mathrm{MHz}$ in the period 11:44-11:48 UT, that is, within $4 \mathrm{~min}$, resulting in a drift rate of $-12.5 \mathrm{kHz} / \mathrm{s}$. The structure occurring on 11:46:40 UT in the dynamic radio spectrum (see Fig. 1) is selected to determine their typical spectral features. The temporal leading edge of its "tail" drifts from $25 \mathrm{MHz}$ towards $15 \mathrm{MHz}$ within $8 \mathrm{~s}$ leading to a drift rate of $-1.25 \mathrm{MHz} / \mathrm{s}$. In Fig. 1, tail structures are also seen in the frequency range $28-32 \mathrm{MHz}$ indicating the emission in the 25-15 MHz range to be the fundamental one. This assumption is supported by the argument that they show a pronounced circular polarization (see Fig. 1) as is typical for the fundamental HB emission (Cairns \& Robinson 1987).

\section{Data analysis}

In the solar corona, the nonthermal radio radiation in the $\mathrm{MHz}$ range is regarded to be plasma emission, that is, the emission of the frequency $f$ takes place near the local electron plasma frequency $\omega_{\mathrm{pe}}=\left(4 \pi \mathrm{e}^{2} N_{\mathrm{e}} / m_{\mathrm{e}}\right)^{1 / 2}\left(e\right.$, elementary charge; $N_{\mathrm{e}}$, electron number density; $m_{\mathrm{e}}$, electron mass) and/or its harmonics (Ginzburg \& Zheleznyakov 1958; Melrose 1985), that is, $f \approx n f_{\mathrm{pe}}\left(f_{\mathrm{pe}}=\omega_{\mathrm{pe}} / 2 \pi\right)$ with $n=1$ and $n=2$ for fundamental and harmonic emission, respectively. Since the electron plasma frequency depends solely on the electron number density $N_{\mathrm{e}}$ and because of the gravitational density stratification of the corona, a radio source causes a negative or positive frequency drift in the dynamic radio spectrum, if it is moving up- or downwards in the corona, respectively. In order to derive a radial source velocity from the frequency drift, a density model of the corona has to be employed. By means of such a density model, a radial distance $r$ from the center of the Sun is related to a definite electron number density and, hence, electron plasma frequency.

The $25 \mathrm{MHz}-\mathrm{level}$ corresponds to an electron number density $N_{\mathrm{e}}=7.75 \times 10^{6} \mathrm{~cm}^{-3}$ assuming fundamental $(n=1)$ emission. We note that the assumption of fundamental emission is justified as already discussed in Sect. 2.

Since the $25 \mathrm{MHz}$ level is probably located in the outer corona (Mann et al. 1999; Warmuth \& Mann 2005; Zucca et al. 2014) the one-fold Newkirk model (1961),

$N_{\mathrm{e}}(r)=N_{0} \times 10^{4.32\left(R_{\odot} / r\right)}$,

with $N_{0}=4.2 \times 10^{4} \mathrm{~cm}^{-3}$ seems to be appropriate in this region. $R_{\odot}$ denotes the radius of the Sun. The Newkirk (1961) model results from white light scattering measurements of the corona and describes the radial behavior of the electron number density very well in the middle of the corona (Koutchmy 1994). It corresponds to a hydrostatic (or barometric) density model with a temperature $T=1.4 \times 10^{6} \mathrm{~K}$ (Mann et al. 1999). Employing this model, the 26 and $23 \mathrm{MHz}-$ levels are located at radial distances $r=1.878 R_{\odot}$, and $1.969 R_{\odot}$ from the center of the Sun, respectively. Thus, a distance of $0.091 R_{\odot}$ is covered by the slowly drifting radio source within a period of $240 \mathrm{~s}$ resulting in a radial velocity of $V_{\mathrm{r}, \mathrm{sd}}=264 \mathrm{~km} \mathrm{~s}^{-1}$. The tails are drifting from $25 \mathrm{MHz}$ towards $15 \mathrm{MHz}$ within $8 \mathrm{~s}$. Since the $25 \mathrm{MHz}$ and $15 \mathrm{MHz}-$ levels are located at radial distances $r=1.906 R_{\odot}$ and $2.370 R_{\odot}$ from the center of the Sun according to Eq. (1), respectively, the radial source velocity of the tail is found to be $V_{\mathrm{r}, \text { tail }}=40400 \mathrm{~km} \mathrm{~s}^{-1}$.

At the $25 \mathrm{MHz}$-level, that is, at $1.906 R_{\odot}$, a magnetic field $B$ of $0.58 \mathrm{G}$ is derived by means of

$B(r)=B_{0} \cdot\left[\left(r / R_{\odot}\right)-1\right]^{-1.5}$,

with $B_{0}=0.5 \mathrm{G}$ (Dulk \& McLean 1978). This formula approximately describes the radial behavior of the magnetic field strength above active regions. Hence, one gets an Alfvén speed (for definition see Appendix A) of $423 \mathrm{~km} \mathrm{~s}^{-1}$ and a ratio between the electron plasma frequency $\omega_{\text {pe }}$ and the electron cyclotron frequency $\omega_{\mathrm{ce}}$ of $\omega_{\mathrm{pe}} / \omega_{\mathrm{ce}}=15$ at the $25 \mathrm{MHz}$ level $\left(\omega_{\mathrm{ce}}=e B / m_{\mathrm{e}} c ; c\right.$, velocity of light $)$. For comparison, a sound speed $c_{\mathrm{s}}=\left(\gamma k_{B} T / \tilde{\mu} m_{\mathrm{p}}\right)^{1 / 2}$ (see Appendix A) and a thermal electron velocity $v_{\mathrm{th}, \mathrm{e}}=\left(k_{B} T / m_{\mathrm{e}}\right)^{1 / 2}\left(k_{B}\right.$, Boltzmann's constant; $m_{\mathrm{p}}$, 
proton mass) of $179 \mathrm{~km} \mathrm{~s}^{-1}$ and $4600 \mathrm{~km} \mathrm{~s}^{-1}$ are obtained for a coronal temperature of $T=1.4 \times 10^{6} \mathrm{~K}, \gamma=5 / 3$, and $\tilde{\mu}=0.6$ (Priest 1982), respectively. With such values for the sound and Alfvén speed, a plasma beta of 0.2 is obtained according to Eq. (A11).

\section{Plasma emission}

As already mentioned in the previous section, the radio emission of the fine structures is assumed to be plasma emission. In this case, energetic electrons excite electrostatic high-frequency plasma waves such as, for example, Langmuir waves which are converted into escaping radio waves by nonlinear plasma processes (Melrose 1985). Hence, the appearance of energetic electrons is a necessary condition for nonthermal radio emission.

Langmuir waves with the frequency $\omega_{\mathrm{L}}$ and wave number $k$ are governed by the dispersion relation

$\omega_{\mathrm{L}}=\sqrt{\omega_{\mathrm{pe}}^{2}+3 k^{2} v_{\mathrm{th}, \mathrm{e}}^{2}}$,

(Baumjohann \& Treumann 1997). These waves can be excited by electron-beam plasma instability. The resonance condition,

$0=\omega_{\mathrm{L}}-V_{\mathrm{b}} k \pm \omega_{\mathrm{ce}} \approx \omega_{\mathrm{L}}-V_{\mathrm{b}} k$,

(Treumann \& Baumjohann 1997) leads to

$V_{\mathrm{b}}=v_{\text {th,e }} \cdot \sqrt{\frac{3 \omega_{\mathrm{L}}^{2}}{\omega_{\mathrm{L}}^{2}-\omega_{\mathrm{pe}}^{2}}}>\sqrt{3} \cdot v_{\text {th,e }}$.

Here, $\omega_{\text {pe }} / \omega_{\text {ce }} \gg 1$ (see Sect. 3 ) has been taken into account. $V_{\mathrm{b}}$ denotes the velocity of the electron beam along the ambient magnetic field. The inspection of Eq. (5) shows, that $V_{\mathrm{b}}>3^{1 / 2} \cdot v_{\text {th,e }}$ is a necessary condition for the excitation of Langmuir waves by an electron beam.

In the case discussed here, the electron velocity distribution function is composed of background electrons with a particle number density $N_{0}$ and temperature $T_{0}$ and the beam electrons with a particle number density $N_{\mathrm{b}}$ and the temperature $T_{\mathrm{b}}$; it can be written for the complete electron velocity distribution function

$$
\begin{aligned}
F(V)= & \frac{1}{\left(N_{0}+N_{\mathrm{b}}\right)} \times\left(\frac{N_{0}}{\sqrt{2 \pi v_{\mathrm{th}, 0}^{2}}} \cdot \mathrm{e}^{-\left(V-V_{0}\right)^{2} / 2 v_{\mathrm{th}, 0}^{2}}\right. \\
& \left.+\frac{N_{\mathrm{b}}}{\sqrt{2 \pi v_{\mathrm{th}, \mathrm{b}}^{2}}} \cdot \mathrm{e}^{-\left(V-V_{\mathrm{b}}\right)^{2} / 2 v_{\mathrm{th}, \mathrm{b}}^{2}}\right)
\end{aligned}
$$

with $v_{\text {th, } 0}=\left(k_{B} T_{0} / m_{\mathrm{e}}\right)^{1 / 2}$ and $v_{\text {th,b }}=\left(k_{B} T_{\mathrm{b}} / m_{\mathrm{e}}\right)^{1 / 2}$. We note that the velocity distribution function is normalized to unity here. The requirement of a current-free plasma leads to $V_{0}=-V_{\mathrm{b}}\left(N_{\mathrm{b}} / N_{0}\right)$. Now, all velocities are normalized to the thermal speed of the background electrons $v_{\mathrm{th}, 0}$, that is, $U=V / v_{\mathrm{th}, 0}, U_{0}=V_{0} / v_{\mathrm{th}, 0}$, and $U_{\mathrm{b}}=V_{\mathrm{b}} / v_{\text {th, }, 0}$. Taking into account $\mathrm{d} V=v_{\mathrm{th}, 0} \mathrm{~d} U$, Eq. (6) can be rewritten as

$F(U)=\frac{1}{(1+v)} \cdot \frac{1}{\sqrt{2 \pi}} \cdot\left(\mathrm{e}^{-\left(U-v U_{\mathrm{b}}\right)^{2} / 2}+\frac{v}{w} \cdot \mathrm{e}^{-\left(U-U_{\mathrm{b}}\right)^{2} / 2 w^{2}}\right)$,

with $v=N_{\mathrm{b}} / N_{0}$ and $w=v_{\text {th, }} / v_{\text {th }, 0}$. Thus, $w$ denotes the width of the beam in velocity space. The occurrence of an instability for exciting Langmuir waves requires that there is a region where the velocity distribution function fulfills $\mathrm{d} F / \mathrm{d} U>0$ (Treumann \& Baumjohann 1997) leading to

$v \cdot \frac{\left(U_{\mathrm{b}}-U\right)}{w^{3}} \cdot \mathrm{e}^{-\left(U-U_{\mathrm{b}}\right)^{2} / 2 w^{2}}>\left(U+v U_{\mathrm{b}}\right) \cdot \mathrm{e}^{-\left(U+v U_{\mathrm{b}}\right)^{2} / 2}$,

by means of Eq. (7). Equation (8) shows that instability occurs only for $U<U_{\mathrm{b}}$. The left-hand side of Eq. (8) has a maximum at $U=U_{\mathrm{b}}-w$. Inserting $U=U_{\mathrm{b}}-w$ into Eq. (8), the inequality,

$v>w^{2} \cdot \mathrm{e}^{1 / 2} \cdot\left[(1+v) U_{\mathrm{b}}-w\right] \cdot \mathrm{e}^{-\left[(1+v) U_{\mathrm{b}}-w\right]^{2} / 2}$,

results in a sufficient condition for exciting Langmuir waves.

\section{Shock drift acceleration}

Here, we assume that the electron beams related to the discussed fine structures (HBs) are produced by SDA. A fast magnetosonic shock wave is accompanied by a magnetic field compression. Thus, it is a moving magnetic mirror, at which charged particles can be reflected and accelerated. The acceleration happens by the electric field, which is induced in the shock transition region.

For a brief introduction to SDA, we refer to the papers by Holman \& Pesses (1983), Ball \& Melrose (2001), and Mann \& Klassen (2005) for a much more detailed description.

Since the tail's associated electron beams have typical velocities $\approx 40000 \mathrm{~km} \mathrm{~s}^{-1}$ (or energies $\approx 5 \mathrm{keV}$ ), they can be treated in a non-relativistic manner.

The SDA is usually described in the de Hoffmann-Teller frame, in which the shock wave is at rest and the motional electric field is removed. Therefore, the reflection process can be treated by conservation of the kinetic energy and the magnetic moment in this special frame. As a result, the velocity gain due to SDA is given by

$V_{\mathrm{r}, \|}=2 v_{\mathrm{s}} \sec \theta-V_{i, \|}$,

with $v_{\mathrm{s}}$ being the shock speed in the laboratory frame (Holman \& Pesses 1983). Here, $V_{i, \|}$ and $V_{\mathrm{r}, \|}$ denote the particle velocity parallel to the ambient magnetic field before and after the reflection process, respectively. $\theta$ is the angle between the upstream magnetic field $\boldsymbol{B}_{1}$ and the shock normal $\boldsymbol{n}_{\mathrm{s}}$. Because of the conservation of the magnetic moment, the particle velocity perpendicular to the magnetic field stays unchanged during the reflection process.

$V_{\mathrm{r}, \perp}=V_{i, \perp}$.

Assuming a Maxwellian distribution (it is normalized to unity here),

$f\left(V_{\|}, V_{\perp}\right)=\frac{1}{\left(2 \pi v_{\mathrm{tn}, \mathrm{e}}^{2}\right)^{3 / 2}} \cdot \mathrm{e}^{-\left(V_{\|}^{2}+V_{\perp}^{2}\right) / 2 v_{\mathrm{the}}^{2}}$,

which is the initial state in the upstream region, after the reflection process, the velocity distribution function of the accelerated electrons has the form (see Eq. (15) in Mann \& Klassen 2005)

$$
\begin{aligned}
f_{\text {acc }}= & \Theta\left(V_{\|}-V_{\mathrm{s}}\right) \cdot \Theta\left(V_{\perp}-V_{\perp, \mathrm{lc}}\right) \\
& \times \frac{1}{\left(2 \pi v_{\mathrm{th}, \mathrm{e}}^{2}\right)^{3 / 2}} \cdot \exp \left(-\frac{\left[\left(V_{\|}-2 V_{\mathrm{s}}\right)^{2}+V_{\perp}^{2}\right]}{2 v_{\mathrm{th}, \mathrm{e}}^{2}}\right),
\end{aligned}
$$

with

$V_{\perp, \mathrm{lc}}=\tan \alpha_{\mathrm{lc}} \cdot \sqrt{\left(V_{\|}-V_{\mathrm{s}}\right)^{2}+V_{\mathrm{e}}^{2}}$, 


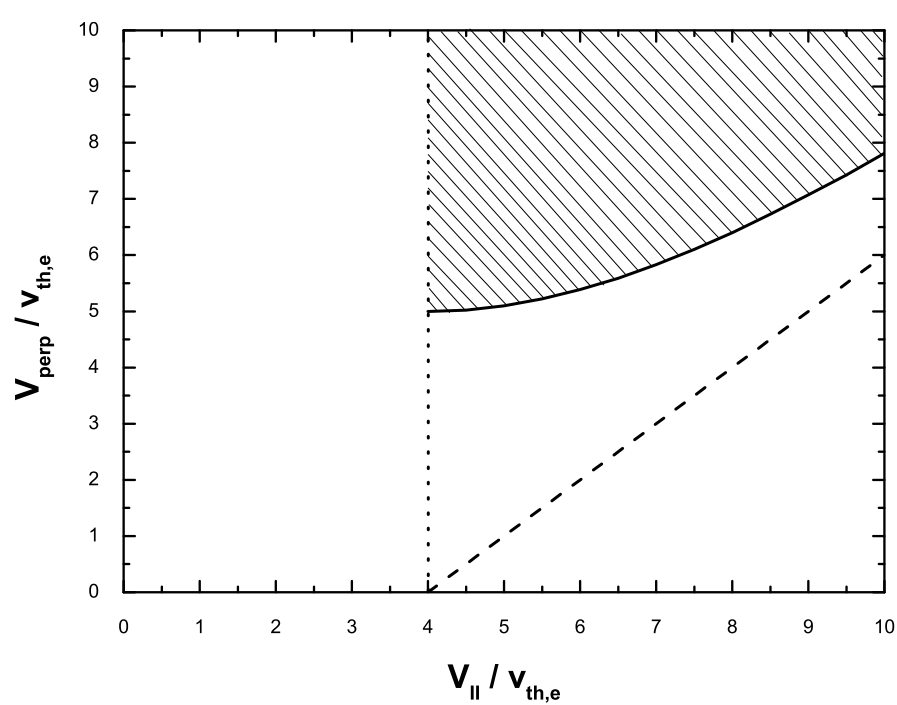

Fig. 3. The velocity distribution function of the accelerated electrons is non-zero in the hatched region in the $V_{\perp}-V_{\|}$-plane for $V_{\mathrm{S}}=4 v_{\text {th,e }}$, $V_{\mathrm{e}}=5 v_{\mathrm{th}, \mathrm{e}}$ and $\alpha_{\mathrm{lc}}=45^{\circ}$. The dashed line represents the line $V_{\perp}=$ $\left(V_{\|}-V_{\mathrm{s}}\right) \cdot \tan \alpha_{\mathrm{lc}}$. The full line represents the function $V_{\perp}=V_{\perp . \mathrm{lc}}\left(V_{\|}\right)$for the above given parameters.

where $V_{\mathrm{s}}=v_{\mathrm{s}} \sec \theta$ and $V_{\mathrm{e}}=\left(2 e \phi_{\mathrm{HT}} / m_{\mathrm{e}}\right)^{1 / 2}$. Here, $\Theta$ is the wellknown step function, that is, $\Theta(x)=1$ for $x \geq 0$ and $\Theta(x)=0$ for $x<0$. We note that $V_{\mathrm{s}}$ represents the shock speed in the de Hoffmann-Teller frame. The loss-cone angle $\alpha_{\mathrm{lc}}$ is given by $\alpha_{\mathrm{lc}}=\arcsin \left[\left(B_{1} / B_{2}\right)^{1 / 2}\right]$ with $B_{1}$ and $B_{2}$ as the magnetic field strength in the up- and downstream regions, respectively.

Due to the different inertia of electrons and protons, a cross-shock potential is established at the shock transition region (Goddrich \& Scudder 1984; Kunic et al. 2001). In the de Hoffmann-Teller frame it is given by

$e \phi_{\mathrm{HT}}=\frac{\gamma}{(\gamma-1)} \cdot k_{B} T_{1} \cdot\left[\frac{T_{2}}{T_{1}}-1\right]$,

where $\gamma$ is the ratio of the specific heats (see Eq. (10) in Mann \& Klassen 2005), and $T_{1}$ and $T_{2}$ denote the temperatures in the upand downstream regions, respectively.

The distribution function of Eq. (13) represents a so-called shifted loss-cone distribution (Leroy \& Mangeney 1984; Wu 1984). It is nonzero in the region defined by $V_{\|} \geq V_{\mathrm{s}}$ and $V_{\perp} \geq V_{\perp, \text { lc }}$ in the $V_{\perp}-V_{\|}$plane as illustrated in Fig. 3 . The reduced distribution function is defined by

$F\left(V_{\|}\right)=2 \pi \cdot \int_{0}^{\infty} \mathrm{d} V_{\perp} \cdot V_{\perp} \cdot f\left(V_{\|}, V_{\perp}\right)$,

with $\mathrm{f}$ as the usual distribution function in the whole velocity space. For the accelerated electrons it is found to be

$F_{\text {acc }}=\Theta\left(V_{\|}-V_{\mathrm{s}}\right) \cdot F_{\mathrm{b}} \cdot \exp \left(-\frac{\left[V_{\|}-V_{\mathrm{s}}\left(1+\cos ^{2} \alpha_{\mathrm{lc}}\right)\right]^{2}}{2 v_{\mathrm{th}, \mathrm{e}}^{2} \cos ^{2} \alpha_{\mathrm{lc}}}\right)$,

with

$F_{\mathrm{b}}=\frac{1}{\left(2 \pi v_{\mathrm{th}, \mathrm{e}}^{2}\right)^{1 / 2}} \cdot \mathrm{e}^{-\left[V_{\mathrm{s}}^{2} \sin ^{2} \alpha_{\mathrm{lc}}+V_{\mathrm{e}}^{2} \tan ^{2} \alpha_{\mathrm{lc}}\right] / 2 v_{\mathrm{the}}^{2},}$

(see Eq. (16) in Mann \& Klassen 2005). The reduced distribution function depends solely on the velocity component $V_{\|}$. For illustration, the shape of the reduced distribution (see Eq. (17)) is presented in Fig. 4; it represents a distribution function of

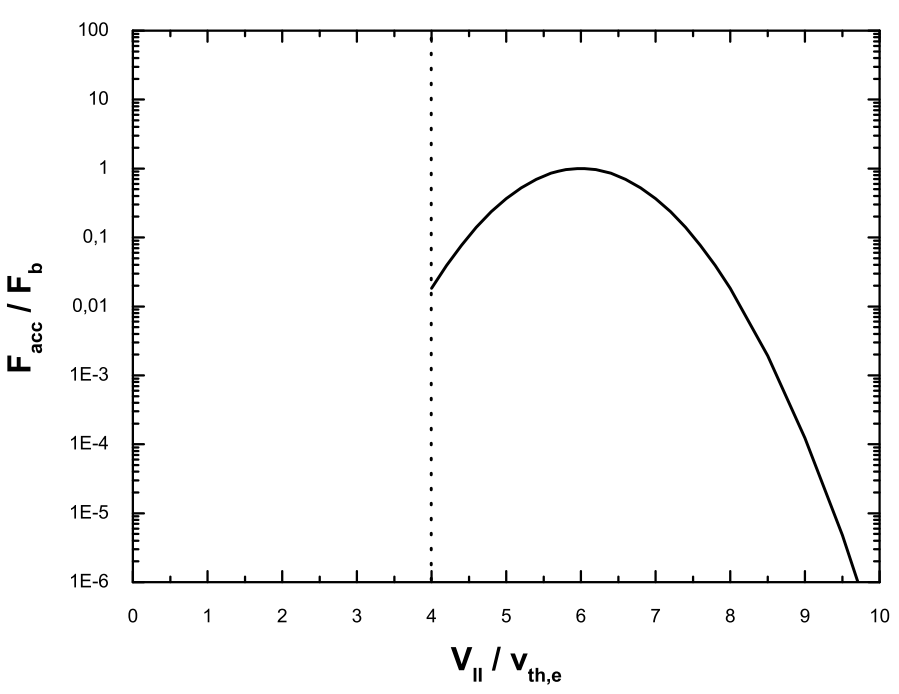

Fig. 4. Shape of the reduced distribution function for $V_{\mathrm{S}}=4 v_{\text {th,e }}, V_{\mathrm{e}}=$ $5 v_{\text {th,e }}$ and $\alpha_{\mathrm{lc}}=45^{\circ}$. Its beam-like shape is evident.

an electron beam with the velocity $V_{\mathrm{b}}=V_{\mathrm{s}}\left(1+\cos ^{2} \alpha_{\mathrm{lc}}\right)$ and the width $2^{1 / 2} v_{\text {th,e }} \cos \alpha_{\text {lc }} . F_{\text {acc }}$ is not vanishing for $V_{\|} \geq V_{\mathrm{s}}$. At $V_{\|}=V_{\mathrm{s}}$, it takes the value

$F_{\mathrm{acc}}\left(V_{\|}=V_{\mathrm{s}}\right)=\frac{1}{\left(2 \pi v_{\mathrm{th}, \mathrm{e}}^{2}\right)^{1 / 2}} \cdot \mathrm{e}^{-\left[V_{\mathrm{s}}^{2}\left(1+\sin ^{2} \alpha_{\mathrm{lc}}\right)+V_{\mathrm{e}}^{2} \tan ^{2} \alpha_{\mathrm{lc}}\right] / 2 v_{\mathrm{th}, \mathrm{e}}^{2}}$.

Once again, the distribution function of the accelerated electrons has the shape of a shifted loss-cone distribution. There are regions with $\partial f_{\text {acc }} / \partial V_{\|}>0$ and $\partial f_{\text {acc }} / \partial V_{\perp}>0$, which gives rise to an instability as a mixture of both a loss-cone and beam instability. As already discussed in Sect. 3, such instabilities lead to exciting Langmuir waves (Treumann \& Baumjohann 1997), which are nonlinearly converted into radio waves. Hence, the electron beams produced by SDA are able to emit radio waves.

\section{Discussion}

As already mentioned in Sect. 1, the chain of fine structures in Fig. 1 is interpreted as the radio signature of a chain of electron beams generated by a shock wave traveling outwards in the corona. Hence, $V_{\mathrm{b}}=V_{\mathrm{s}}\left(1+\cos ^{2} \alpha_{\mathrm{lc}}\right)$ and $v_{\mathrm{s}}$ can be interpreted as the velocity of the electron beam and the associated shock, respectively.

Because the shock-accelerated electron beams must be able to excite Langmuir waves in order to emit radio waves, their velocity $V_{\mathrm{b}}$ must necessarily fulfill $V_{\mathrm{b}}>3^{1 / 2} v_{\text {th,e }}$ (see Eq. (5)) leading to the requirement

$M_{\mathrm{A}}=\frac{V_{\mathrm{s}}}{v_{\mathrm{A}}}>\sqrt{3} \cdot \frac{v_{\mathrm{th}, \mathrm{e}}}{v_{\mathrm{A}}} \cdot \frac{1}{\left(1+\cos ^{2} \alpha_{\mathrm{lc}}\right)}=\frac{18.9}{\left(1+\cos ^{2} \alpha_{\mathrm{lc}}\right)}$.

Here, $v_{\text {th,e }}=4600 \mathrm{~km} \mathrm{~s}^{-1}$ and $v_{\mathrm{A}}=423 \mathrm{~km} \mathrm{~s}^{-1}$ (see Sect. 3 ) have been used for the thermal electron speed $v_{\text {th,e }}$ and Alfvén speed $v_{\mathrm{A}}$, respectively. As a result, the appearance of radio emission from shock-accelerated electron beams requires that the associated shock must be a high Alfvén-Mach number (i.e., $M_{\mathrm{A}} \gg 1$ ) in the de Hoffmann-Teller frame and, hence, a nearly perpendicular one (i.e., $\theta \rightarrow 90^{\circ}$ ). That is the reason why the RankineHugoniot relationships are especially evaluated for the case of an almost perpendicular shock in Appendix A, that is, in the regime $M_{\mathrm{A}} \gg 1$ and $\theta \rightarrow 90^{\circ}$. We note that the relationship between the quantities of the up- and downstream regions of the shock 


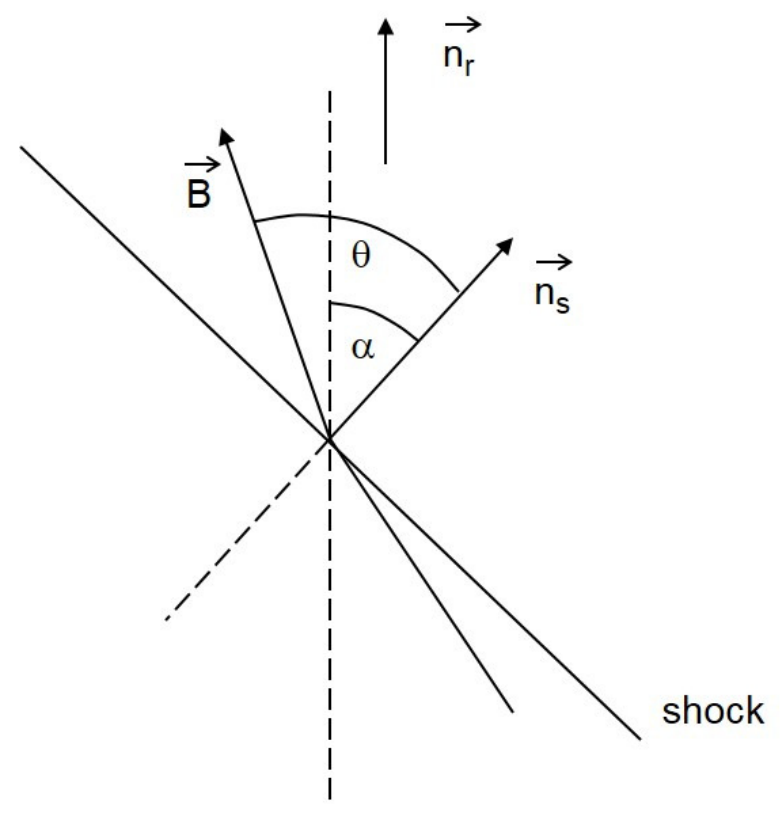

Fig. 5. Shock geometry in the corona.

can be studied by the magnetohydrodynamic Rankine-Hugoniot relationships in a magnetized plasma such as the corona (see Appendix A).

Figure 5 presents the shock geometry in the corona. The shock is oriented perpendicular to the image plane. The shock normal $\boldsymbol{n}_{\mathrm{s}}$ takes an angle $\alpha$ to the radial direction $\boldsymbol{n}_{\mathrm{r}}$ and an angle $\theta$ to the upstream magnetic field $\boldsymbol{B}_{1}$.

According to Fig. 5, the radial velocities of the shock $v_{\mathrm{r}, \mathrm{s}}$ and of the electron beam $V_{\mathrm{r}, \mathrm{b}}$ are given by $v_{\mathrm{r}, \mathrm{s}}=v_{\mathrm{s}} \cdot \cos \alpha$ and $V_{\mathrm{r}, \mathrm{b}}=V_{\mathrm{b}} \cdot \cos (\theta-\alpha)$, respectively. They are identified with the velocities of the slowly drifting chain, that is, $V_{\mathrm{r}, \mathrm{sd}}=v_{\mathrm{r}, \mathrm{s}}$, and of the tails, that is, $V_{\mathrm{r} \text {,tail }}=V_{\mathrm{r}, \mathrm{b}}$, as derived from the dynamic radio spectrum (see Fig. 1).

Then, the real shock speed is given by $v_{\mathrm{s}}=v_{\mathrm{r}, \mathrm{s}} / \cos \alpha$ resulting in

$\cos \alpha=\frac{v_{\mathrm{r}, \mathrm{s}}}{v_{\mathrm{A} 1}} \cdot \frac{1}{M_{\mathrm{A}}^{*}}=\frac{0.62}{M_{\mathrm{A}}^{*}}$,

with $M_{\mathrm{A}}^{*}=v_{\mathrm{s}} / v_{\mathrm{A} 1}$ as the Alfvén-Mach number in the laboratory frame. Here, $v_{\mathrm{r}, \mathrm{s}}=V_{\mathrm{r}, \mathrm{sd}}=264 \mathrm{~km} \mathrm{~s}^{-1}$ and $v_{\mathrm{A} 1}=463 \mathrm{~km} \mathrm{~s}^{-1}$ have been used. Thus, the angle $\alpha$ and the shock speed $v_{\mathrm{s}}=M_{\mathrm{A}}^{*} \cdot v_{\mathrm{A} 1}$ can be determined for a given value of $M_{\mathrm{A}}^{*}$. Furthermore, the relationship

$$
\begin{aligned}
\frac{V_{\mathrm{r}, \text { tail }}}{V_{\mathrm{r}, \mathrm{sd}}} & =153=\frac{V_{\mathrm{b}} \cdot \cos (\theta-\alpha)}{v_{\mathrm{s}} \cdot \cos \alpha} \\
& =[1+\tan \alpha \cdot \tan \theta] \cdot\left(1+\cos ^{2} \alpha_{\mathrm{lc}}\right) \\
& =[1+\tan \alpha \cdot \tan \theta] \cdot\left(2-\frac{1}{X}\right)
\end{aligned}
$$

can be derived for calculating the angle $\theta$ for a given value of $M_{\mathrm{A}}^{*}$. The angle $\alpha_{\mathrm{lc}}$ is given by $\alpha_{\mathrm{lc}}=\arcsin \left[\left(B_{1} / B_{2}\right)^{1 / 2}\right]=$ $\arcsin \left[X^{-1 / 2}\right]$ for a nearly perpendicular shock (see Eq. (A.9)). We note that the density jump $X$ across the shock is a function of $M_{\mathrm{A}}^{*}$ according to Eq. (A.10). With the so-found angles $\alpha, \theta$, and $\alpha_{\mathrm{lc}}$, the velocity of the electron beam $V_{\mathrm{b}}=v_{\mathrm{s}} \cdot \sec \theta \cdot\left(1+\cos ^{2} \alpha_{\mathrm{lc}}\right)$ can be determined. As a result, all quantities can be fixed for a given value of $M_{\mathrm{A}}^{*}$ or $X$ taking into account the results of the Rankine-Hugoniot relationships (see Appendix). Varying $X$ in the range 1.4-2.6, the best fit with respect to the observations, that is, $V_{\mathrm{r}, \mathrm{sd}}=v_{\mathrm{r}, \mathrm{s}}=264 \mathrm{~km} \mathrm{~s}^{-1}$ and $V_{\mathrm{r}, \mathrm{tail}}=V_{\mathrm{r}, \mathrm{b}}=$ $40400 \mathrm{~km} \mathrm{~s}^{-1}$, is found for $X=2.3$ resulting in: $M_{\mathrm{A}}^{*}=2.38$, $v_{\mathrm{s}}=1010 \mathrm{~km} \mathrm{~s}^{-1}, \alpha=74.8^{\circ}, \alpha_{\mathrm{lc}}=41.3^{\circ}, \theta=87.8^{\circ}$, $V_{\mathrm{s}}=26.500 \mathrm{~km} \mathrm{~s}^{-1}$, and $V_{\mathrm{b}}=41400 \mathrm{~km} \mathrm{~s}^{-1}$. This leads to an Alfvén-Mach number of the associated shock $M_{\mathrm{A}}=V_{\mathrm{s}} / v_{\mathrm{A}}=63$ in the de Hoffmann-Teller frame as should be expected (i.e., $M_{\mathrm{A}} \gg 1$; see the discussion with respect to Eq. (20)). Employing all these parameters, the radial velocity of the electron beam $V_{\mathrm{r}, \mathrm{b}}=V_{\mathrm{b}} \cdot \cos (\theta-\alpha)$ is $40400 \mathrm{~km} \mathrm{~s}^{-1}$ and agrees well with the radial speed of the tail's radio source as derived from the dynamic radio spectra in Fig. 1. Thus, the cycle of argumentation is closed in the best way for this fit. Consequently, it is justified to consider the derived shock parameters as the real ones.

By means of SDA the electrons are accelerated up to velocities $V_{\|} \geq V_{\mathrm{s}}$. We note that both $V_{\mathrm{s}}$ and $V_{\mathrm{b}}$ are greater than $3^{1 / 2} v_{\text {th,e }}$ (i.e., $V_{\mathrm{s}}=26500 \mathrm{~km} \mathrm{~s}^{-1}>5.8 v_{\text {th,e }}$ and $V_{\mathrm{b}}=41400 \mathrm{~km} \mathrm{~s}^{-1}$ $>9.0 v_{\text {th,e }}$ with $v_{\text {th,e }}=4600 \mathrm{~km} \mathrm{~s}^{-1}$ ) as required for exciting Langmuir waves (see the discussion in Sect. 4). The ratio between the number density of the beam electrons and background ones $N_{\mathrm{b}} / N_{0}$ can be determined to be $v=N_{\mathrm{b}} / N_{0}=F_{\mathrm{b}} \cdot v_{\text {th,e }}^{1 / 2}$ by comparing the velocity distribution function of shock-accelerated electrons (see Eq. (17)) with that of an electron beam (see Eq. (6)). $F_{\mathrm{b}}$ is given by Eq. (18). With the above given parameters, one gets $v=N_{\mathrm{b}} / N_{0}=4.9 \times 10^{-11}$ with $V_{\mathrm{e}}=6.54 v_{\text {th,e }}$ according to Eq. (A.13). Then, the sufficient condition for exciting Langmuir waves (see Eq. (9)) is well fulfilled by the shock-accelerated electrons, that is, $v=4.9 \times 10^{-11}>1.3 \times 10^{-14}$.

In the special case of $V_{\|} \approx V_{\mathrm{s}}$, the accelerated electrons are at rest in the de Hoffmann-Teller frame, or, in other words, they are attached at the shock, that is, they cannot leave the shock front. The inspection of Eqs. (13) and (17) as well as of Fig. 4 reveals that there is a substantial number of accelerated electrons at velocities of $V_{\|} \approx V_{\mathrm{s}}$. They show a positive slope in the velocity distribution (see Eq. (17) and Fig. 4), that is, $\mathrm{d} F_{\text {acc }} / \mathrm{d} V_{\|}>0$, giving rise to Langmuir wave excitation (Treumann \& Baumjohann 1997). Since they cannot leave the shock front, they move with the shock through the corona. These electrons could cause the radio radiation of the heads, whereas the tails are generated by the electrons forming the beam at velocities $V_{\|} \approx V_{\mathrm{b}}$. Thus, this approach is also able to explain the head and tail radiation as different components of the fine structures.

To emit radio waves via plasma emission, the excitation of Langmuir waves is necessary. As discussed in Sect. 4, the velocity of the electron beam generating Langmuir waves must exceed at least $3^{1 / 2} v_{\text {th,e, }}$, that is,

$v_{\mathrm{s}} \sec \theta\left(1+\cos ^{2} \alpha_{\mathrm{ls}}\right)>\sqrt{3} \cdot v_{\text {th,e }}$.

This condition is dependent on the shock speed $v_{\mathrm{s}}$ and the thermal electron velocity $v_{\text {th,e }}$ as well as the angles $\theta$ and $\alpha_{\text {lc }}$. The angle $\theta$ is the most crucial parameter. The condition (23) is well fulfilled for $\theta>87^{\circ}$ in the case discussed here. Once again, $\theta$ is the angle between the shock normal and the upstream magnetic field. This angle can vary during the motion of the shock through the corona. If the value of $\theta$ drops below this threshold, the excitation of Langmuir waves and, consequently, the associated radio emission are switched off. Thus, the appearance of the discussed fine structures is crucially dependent on the value of $\theta$. This could explain the bursty occurrence of them leading to a slowly drifting chain of such structures in the dynamic radio spectrum. 


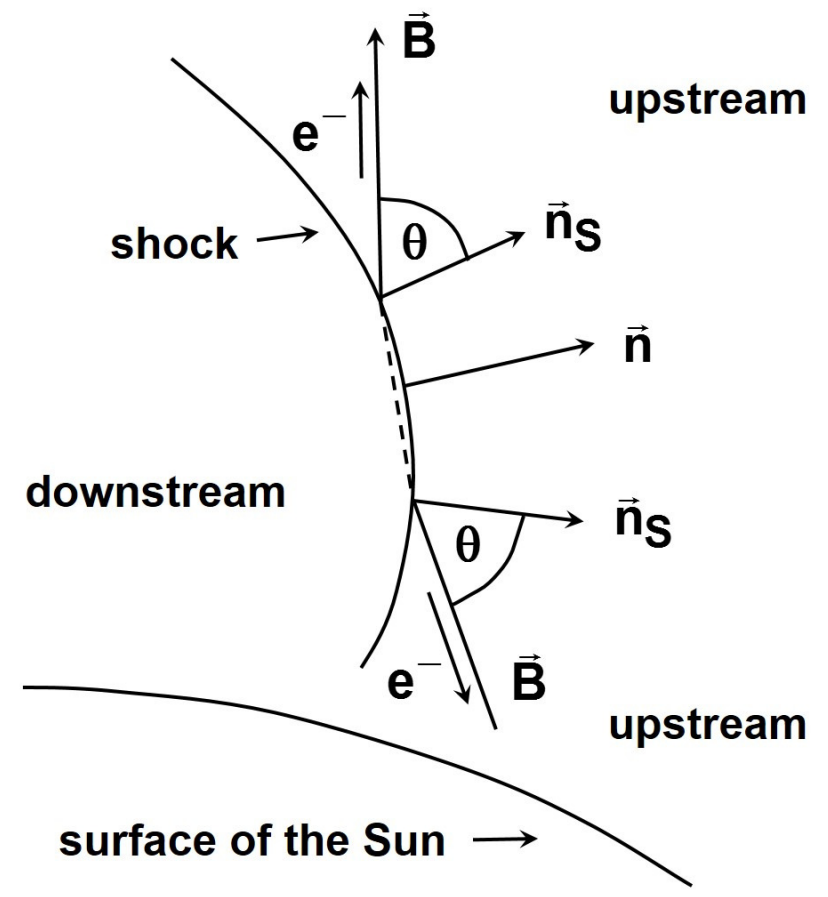

Fig. 6. Sketch of a shock propagating laterally with respect to the solar surface in the corona. The shock is traveling into the direction $\boldsymbol{n}$. At the flanks, shocks are formed with different angles $\theta$ between the shock normal $\boldsymbol{n}_{\mathrm{s}}$ and the upstream magnetic field $\boldsymbol{B}$. Electrons accelerated due to SDA can propagate along the upstream magnetic field towards both the higher and/or lower corona.

Shock drift acceleration only produces electron beams in the upstream region. Such beams are necessary to excite Langmuir waves and, consequently, for the emission of radio waves, as already mentioned in Sect. 1. Herring bones drift away from the backbone towards low and high frequencies in dynamic radio spectra. This phenomenon was interpreted by Holman \& Pesses (1983) as a curved shock wave traveling through the corona. It can generate electron beams at different parts of the shock as illustrated in Fig. 6. That leads to a drift towards lower and/or higher frequencies of the associated HBs in dynamic radio spectra. But the emission of radio waves can only happen if Langmuir waves are excited, which requires the fulfilment of condition (23). If this condition is not fulfilled, for example in the case discussed where $\theta<87^{\circ}$, then there is no radio emission. That may explain why only negatively drifting tails are seen in the observations discussed here.

Energetic electrons in the solar corona are also observed in terms of type-III bursts in the solar radio radiation (Wild \& McCready 1950; Reid \& Ratcliffe 2014, for review). They appear as emission stripes rapidly drifting from high to low frequencies (sometimes down to a few tens of $\mathrm{kHz}$ ) in dynamic radio spectra (Suzuki \& Dulk 1985). These bursts are regarded as the radio signature of beams of energetic electrons traveling along magnetic field lines through the corona and sometimes up to the interplanetary space. These energetic electrons are produced as a result of flares (or magnetic reconnection). Initially, they have a power law distribution in velocity space. Due to their spatio-temporal evolution, they form a beam-like distribution, that is, with positive slope in the velocity distribution, after a distance from the initial acceleration region as discussed by Reid et al. (2011, 2014). Then, they are able to excite Langmuir waves which are converted into radio waves by nonlinear wave-particle interactions (Melrose 1985). In contradiction to the energetic electrons associated with type-III bursts, SDA initially generates a beam-like electron distribution (see Eq. (17)). Thus, the excitation of Langmuir waves and the emission of radio waves in terms of HBs happens immediately in the vicinity of the shock. In the case discussed here (see Fig. 1), the tails appear in the frequency range $25-15 \mathrm{MHz}$, that is, the associated electron beam travels over a distance of $323 \mathrm{Mm}$ before the radio emission is switched off. According to the discussion by Reid \& Kontar $(2013,2015)$, such electron beams are influenced by Coulomb collisions, scattering at density fluctuation in the background plasma, and inhomogeneities of the magnetic field as well as emissions and absorptions of Langmuir waves. Because they have energies of about $5 \mathrm{keV}$, as in the present case, Coulomb collisions and scattering at density fluctuations could be the most important influences on the electron beam (see the discussion in Mann \& Klassen 2005; and the results of Reid \& Kontar 2013, 2015) resulting in a destruction of the beam followed by the interruption of Langmuir wave excitation, and, finally, in the switching off of the radio emission.

As a result, the HB structures observed in the decameter range are generated by electron beams produced by a nearly perpendicular shock, which laterally propagates in the corona. Such shocks can be driven by the expanding flanks of CMEs moving upwards through the corona. Such a scenario is confirmed by observations (Sala-Matamoros et al. 2016), theoretical studies (Schmidt \& Cairns 2012a, b) and numerical simulations (Schmidt et al. 2014).

\section{Summary}

The Ukrainian radio telescope URAN-2 recorded special fine structures in the solar radio radiation on April 7, 2011. They appear as slowly drifting chains of stripes of enhanced radio emission in the dynamic radio spectrum in the frequency range 8-33 MHz (see Fig. 1). The chain drifts from 26 to $23 \mathrm{MHz}$ within $240 \mathrm{~s}$. The discussed fine structures consist of two components, namely, the head at the high frequency edge and the tail, which rapidly drifts from high to low frequencies, that is, over $\approx 10 \mathrm{MHz}$ within $\approx 8 \mathrm{~s}$. The slow drift rate of the chains corresponds to those of solar type-II radio bursts in this frequency range. Solar type-II radio bursts are regarded as the radio signature of shock waves traveling outwards through the corona. The tails resembling HBs appear as special fine structures in solar type-II radio bursts. Herringbones are considered to be the radio signatures of electron beams accelerated at the type-II-burstassociated shock wave. For these reasons, the tails are interpreted as shock-accelerated electron beams. According to this view, the slow drift is identified as a shock wave moving outwards in the corona. As mentioned in Sect. 1, there are also cases in which the tails drift towards higher frequencies. These can be explained in the following way: generally, in the case of SDA, the electrons are scattered back into the upstream region, where they appear as beams of energetic electrons. If the upstream magnetic field is directed outwards, that is, into regions with lower electron number densities, the tails show a negative drift in the dynamic radio spectrum. In the opposite case, in which the upstream magnetic field is directed towards the lower corona, that is, into regions with higher electrons number densities, the tails drift towards higher frequencies.

Since the radio emission in this frequency range, that is, 8-33 MHz, is thought to be plasma emission (as discussed in Sect. 4), these radio waves are emanated from the outer corona. The radial velocities of the shock-accelerated electron beams 
and the associated shock waves are derived to $40400 \mathrm{~km} \mathrm{~s}^{-1}$ and $264 \mathrm{~km} \mathrm{~s}^{-1}$, respectively.

The electrons are thought to be accelerated by SDA. In the framework of SDA, a shifted loss-cone distribution is established for the accelerated electrons. This results in a beam-like distribution with respect to the electron velocities parallel to the ambient magnetic field. Such a distribution is unstable for exciting Langmuir waves. This requires the velocity of the electron beam to exceed $3^{1 / 2}$ times the thermal electron velocity. If the electron beam is generated by SDA, then only a nearly-perpendicular fast mode shock fulfills this condition. This is why the RankineHugoniot relationships are evaluated under the assumption of an almost perpendicular shock (see Appendix A).

Taking all of the above into account, the most robust scenario linking the observational data, that is, the radial velocities of the shock and the electron beam, to the theoretical results, involves an almost perpendicular shock, traveling with a speed of $1010 \mathrm{~km} \mathrm{~s}^{-1}$ nearly horizontal with respect to the surface of the Sun, generating electron beams with a velocity of $41400 \mathrm{~km} \mathrm{~s}^{-1}$ by means of SDA.

Acknowledgements. A. I. Brazhenko, A. A. Konovalenko, G. Mann, and V. N. Melnik express their thanks to H. O. Rucker and the staff of the Space Research Institute of the Austrian Academy of Sciences (Graz, Austria) for their kind hospitality; work was carried out at this institute. G.M. performed this work under the framework of grant No. 50QL0001 of the Deutsches Zentrum für Luft- und Raumfahrt (DLR). V.N.M. and A.A.K. made this work in the frame of project "Spectr-3" by National Academy of Sciences of Ukraine.

\section{References}

Alvarez, H., \& Haddock, F. T. 1973, Sol. Phys., 29, 197

Aurass, H. 1996, in Coronal Physics from Radio and Space Observations, ed. G Trottet (Heidelberg: Springer Verlag), Lect. Not. Phys., 135

Ball, L., \& Melrose, D. B. 2001, PASA, 18, 361

Baumjohann, W., \& Treumann, R. A. 1997, Basic Space Plasma Physics, (London: Imperial College Press) 202

Brazhenko, A. I., Bulatsen, V. G., Vashchishin, R. V., et al. 2005, Kinematika i Fizika Nebesnykh Tel, Suppl., 5, 43

Brazhenko, A., Melnik, V., Konovalenko, A., et al. 2012, Proc. European Planetary Science Congress (September 23-28, 2012 in Madrid, Spain), 544 Cairns, I. H., \& Robinson, R. D. 1987, Sol. Phys., 111, 365

Cairns, I. H., Knock, S. A., Robinson, P. A., \& Kunic, Z. 2003, Space Sci. Rev., 107,27

Cane, H. V., \& White, S. M. 1989, Sol. Phys., 120, 137

Dorovskyy, V. V., Melnik, V. N., Konovalenko, A. A., et al. 2015, Sol. Phys., 290, 2031

Dulk, G. A., \& McLean, D. J. 1978, A\&A, 66, 315

Goodrich, C. C., \& Scudder 1984, J. Geophys. Res., 89, 6654

Holman, G. D., \& Pesses, M. E. 1983, ApJ, 267, 837

Koutchmy, S. 1994, Adv. Space Res., 14, 29
Kunic, Z. Cairns, I. H., \& Knock, S. A. 2001, J. Geophys. Res., 107, 101029 Leroy, M. M., \& Mangeney, A. 1984, Ann. Geophys., 2, 449

Magdalenic, J., Marque, C., Zhukov, A. N., Vršnak, B., \& Veronig, A. 2012, ApJ, 746, 152

Mann, G. 1995, in Coronal Magnetic Energy Release, eds. A. O. Benz, \& A Krüger (Heidelberg: Springer Verlag), Lect. Not. Phys. 183

Mann, G. 2006, in Solar Eruptions and Energetic Particles (AGU, Washington (DC)), Geophys. Monograph Series, 165, 221

Mann, G., \& Klassen, A. 2002, Proc. 10th European Solar Physics Meeting, Prague, Czech Rep., ESA SP, 506, 245

Mann, G., \& Klassen, A. 2005, A\&A, 441, 319

Mann, G., Jansen, F., MacDowall, R. J., Kaiser, M. L. \& Stone, R. G. 1999, A\&A, 348, 614

Melnik, V. N., Konovalenko, A. A., Rucker, H. O., et al. 2004, Sol. Phys., 222, 151

Melnik, V. N., Rucker, H. O., Konovalenko, A. A., et al. 2008, in Solar Physics Research Trends, ed. P. Wang (New York: Nova Science Publishers), 287

Melrose, D. 1985, in Solar Radio Physics, eds. D. J. McLean, \& N. R. Labrum (Cambridge: Cambridge Univ. Press), 177

Nelson, G. S., \& Melrose, D. 1985, in Solar Radio Physics, eds. D. J. McLean, \& N. R. Labrum (Cambridge: Cambridge Univ. Press), 333

Newkirk, G. A. 1961, ApJ, 133, 983

Nindos, A., Aurass, H., Klein, K.-L., \& Trottet, G. 2008, Sol. Phys., 253, 3

Priest, E. R. 1982, Solar Magnetohydrodynamics (Dordrecht: Kluwer Academic Publishers), 1999

Reid, H. A. S., \& Kontar, E. P. 2013, Sol. Phys., 285, 217

Reid, H. A. S., \& Kontar, E. P. 2015, A\&A, 577, A124

Reid, H. A. S., \& Ratcliffe, H. 2014, RA\&A, 14, 773

Reid, H. A. S., Vilmer, N., \& Kontar, E. P. 2011, A\&A, 529, A66

Reid, H. A. S., Vilmer, N., \& Kontar, E. P. 2014, A\&A, 567, A85

Ryabov, V. B., Vavriv, D. M., Zarka, P., et al. 2010, A\&A, 510, A16

Salas-Matamoros, C., Klein, K.-L., \& Rouillard, A. P. 2016, A\&A, 590, A135

Schmidt, J. M., \& Cairns, I. H. 2012a, J. Geophys. Res., 117, A04106

Schmidt, J. M., \& Cairns, I. H. 2012b, J. Geophys. Res., 117, A11104

Schmidt, J. M., Cairns, I. H., \& Lobzin, V. V. 2014, J. Geophys. Res., 119, 6042

Smerd, S. F., Wild, J. P., \& Sheridan, K. V. 1962, Austr. J. Phys., 15, 180

Stewart, R. T., Howard, R. A., Hansen, F., Gergely, T., \& Kundu, M. R. 1974a, Sol. Phys., 36, 219

Stewart, R. T., McCabe, M., Koomen, M. J., Hansen, F., \& Dulk, G. 1974b, Sol. Phys., 26, 203

Stewart, R. T., \& Magun, A. 1980, PASA, 3, 375

Suzuki, S., \& Dulk, G. A. 1985, in Solar Radio Physics, eds. D. J. McLean, \& N. R. Labrum (Cambridge: Cambridge Univ. Press), 289

Suzuki, S., Stewart, R. T., \& Magun, A. 1982, in Radikophysics of the Sun, eds. M. R. Kundu, \& T. R. Gergely, IAU Symp., 86

Treumann, R. A., \& Baumjohann, W. 1997, Advance Space Plasma Physics (London: Imperial College Press), 69

Uchida, Y. 1960, PASJ, 12, 376

Uchida, Y., Altschuler, D., \& Newkirk, G. 1973, Sol. Phys., 28, 495

Vršnak, B., Ruždjak, V., \& Aurass, H. 1995, Sol. Phys., 158, 331

Warmuth, A., \& Mann, G. 2005, A\&A, 435, 1123

Wild, J. P., \& McCready, L. L. 1950, Austr. J. Sci. Res., Ser. A, 3, 387

Wild, J. P., \& Smerd, S. F. 1972, ARA\&A, 10, 159

Wu, C. S. 1984, J. Geophys. Res., 89, 8857

Zlobec, P. Messerotti, M., Karlicky, M., \& Urbarz, H. 1993, Sol. Phys., 144, 373

Zucca, P., Carley, E. P., Bloomfield, D. Sh., \& Gallagher, P. T. 2014, A\&A, 564, A47 


\section{Appendix A: Rankine-Hugoniot relationships in magnetohydrodynamics}

A shock wave is basically a discontinuity with a mass flux across it. It is additionally a dissipative structure. In a magnetized plasma such as the corona, shock waves can be described by conservation of mass, momentum, energy, and magnetic flux in terms of the magnetohydrodynamic (MHD) equations. This is usually done in the de Hoffmann-Teller frame, in which the shock is at rest and the motional electric field is removed (see e.g., Priest 1982).

The framework of the shock is given in the following way: The shock normal $\boldsymbol{n}_{\mathrm{s}}$ is directed along the $x$-axis, that is, the shock itself is located in the $y-z$ plane. The magnetic field $\boldsymbol{B}$ is put in the $x-z$ plane and takes an angle $\theta$ to the $x$-axis in the upstream region. All quantities with the subscript 1 and 2 denote those in the up- and downstream regions, respectively. Then, the conservation of mass, $x$ - and $z$-component of the momentum, energy and magnetic flux leads to the jump relationships in terms of $X=\rho_{2} / \rho_{1}$ :

$$
\begin{aligned}
B_{2 x}= & B_{1 x}, \\
\frac{v_{2 x}}{v_{1 x}}= & \frac{1}{X}, \\
\frac{v_{2 z}}{v_{1 z}}= & \frac{M_{\mathrm{A}}^{2}-1}{M_{\mathrm{A}}^{2}-X}, \\
\frac{B_{2 z}}{B_{1 z}}= & \frac{v_{2 z}}{v_{1 z}} \cdot X, \\
\frac{T_{2}}{T_{1}}= & 1+\frac{(\gamma-1)}{2} \cdot \frac{v_{\mathrm{A} 1}^{2}}{c_{\mathrm{s} 1}^{2}} \cdot M_{\mathrm{A}}^{2} \\
& \times\left\{1-\frac{\cos ^{2} \theta}{X^{2}}-\sin ^{2} \theta \cdot \frac{\left(M_{\mathrm{A}}^{2}-1\right)^{2}}{\left(M_{\mathrm{A}}^{2}-X\right)^{2}}\right\},
\end{aligned}
$$

(Priest 1982). Here, the Alfvén and sound speed are given by $v_{\mathrm{A} 1}=B_{1} /\left(4 \pi \rho_{1}\right)^{1 / 2}$ and $c_{\mathrm{s} 1}=\left(\gamma p_{1} / \rho_{1}\right)^{1 / 2}(\gamma$, ratio of the specific heats) in the upstream region, respectively. $\rho$ denotes the mass density, which is related to the full particle number density $N$ by $\rho=\tilde{\mu} m_{\mathrm{p}} N\left(m_{\mathrm{p}}\right.$, proton mass; $\tilde{\mu}$, mean molecular weight $)$. Additionally, the equation of state $p=N k_{B} T$ has been used. It relates the pressure $p$ to the full particle number $N$ and the temperature $T$. Then, the sound speed is also given by $c_{\mathrm{s} 1}=\left(\gamma k_{B} T_{1} / \tilde{\mu} m_{\mathrm{p}}\right)^{1 / 2}$. The Alfvén-Mach number $M_{\mathrm{A}}$ is defined by $M_{\mathrm{A}}=\left(v_{\mathrm{s}} / v_{\mathrm{A} 1}\right) \cdot \sec \theta$; it is actually the Alfvén-Mach number in the de Hoffmann-Teller frame. Here, $v_{\mathrm{s}}$ denotes the shock speed in the laboratory frame. These jump relationships are supplemented by a polynominal of the third order in $X$ :

$$
\begin{aligned}
0= & \left(M_{\mathrm{A}}^{2}-X\right)^{2} \\
& \times\left\{X \frac{c_{\mathrm{s} 1}^{2}}{v_{\mathrm{A} 1}^{2}}+\frac{M_{\mathrm{A}}^{2}}{2} \cos ^{2} \theta[X(\gamma-1)-(\gamma+1)]\right\} \\
& +X \cdot \frac{M_{\mathrm{A}}^{2}}{2} \cdot \sin ^{2} \theta \times \\
& \left\{[\gamma+X(2-\gamma)] M_{\mathrm{A}}^{2}-X[(\gamma+1)-X(\gamma-1)]\right\},
\end{aligned}
$$

(Priest 1982). In the limit $X \rightarrow 1$, this provides the well-known three MHD modes, namely the Alfvén wave $\left(M_{\mathrm{A}}=1\right)$ as well as the fast $\left(M_{\mathrm{A}}>1\right)$ and slow $\left(M_{\mathrm{A}}<1\right)$ magnetoacoustic waves.

By means of Eqs. (A.1), (A.3), and (A.4), one gets

$$
\frac{B_{2}^{2}}{B_{1}^{2}}=\cos ^{2} \theta+\frac{\left(M_{\mathrm{A}}^{2}-1\right)^{2} X^{2}}{\left(M_{\mathrm{A}}^{2}-X\right)^{2}} \cdot \sin ^{2} \theta .
$$

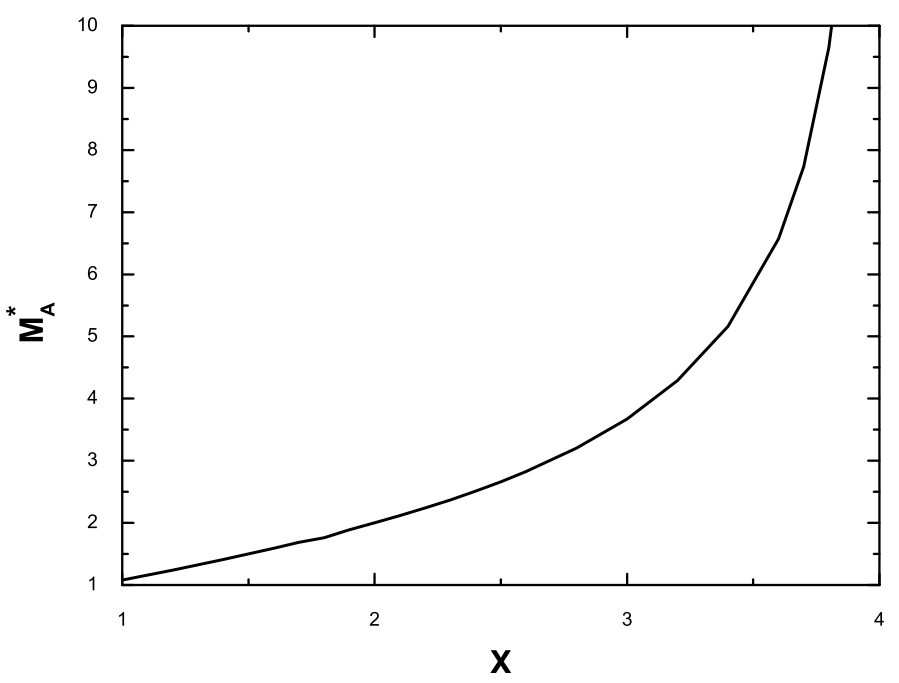

Fig. A.1. Dependence of $M_{\mathrm{A}}^{*}$ on $X$ according to Eq. (A.10) for $\beta=0.2$.

At a shock, there must be a positive jump of the density (i.e., $X>1$ ) in order to guarantee an increase of the entropy (see Priest 1982), which is necessary for a dissipative structure. Then, according to Eq. (A.7), $B_{1}<B_{2}$, that is, magnetic field energy increases across the fast-mode shocks (i.e., $M_{\mathrm{A}}>X$ ).

In the special case of a nearly perpendicular shock, that is, $M_{\mathrm{A}} \gg 1$ and $\theta \rightarrow 90^{\circ}$, Eqs. (A.5) and (A.7) reduce to

$\frac{T_{2}}{T_{1}}=1+\frac{(\gamma-1)}{2} \cdot \frac{v_{\mathrm{A} 1}^{2}}{c_{\mathrm{s} 1}^{2}} \cdot\left(M_{\mathrm{A}}^{*}\right)^{2} \cdot\left(1-\frac{1}{X^{2}}\right)$,

and

$\frac{B_{2}}{B_{1}}=X$,

with $M_{\mathrm{A}}^{*}=v_{\mathrm{s}} / v_{\mathrm{A} 1}$. In this limit, the polynominal (A.6) provides

$\left(M_{\mathrm{A}}^{*}\right)^{2}=X \cdot \frac{\gamma \beta+[\gamma+X(2-\gamma)]}{[(\gamma+1)-X(\gamma-1)]}$.

Here, $\beta$ denotes the ratio between the thermal and the magnetic pressure, that is,

$\beta=\frac{8 \pi}{B^{2}} \cdot N k_{B} T=\frac{2 c_{\mathrm{s} 1}^{2}}{\gamma v_{\mathrm{A} 1}^{2}}$.

The inspection of Eq. (A.10) shows $\left(M_{\mathrm{A}}^{*}\right)^{2} \rightarrow \infty$ if $X \rightarrow$ $(\gamma+1) /(\gamma-1)(=4$ for $\gamma=5 / 3)$. The dependence of $M_{\mathrm{A}}^{*}$ on $X$ according to Eq. (A.10) is illustrated for $\beta=0.2$ in Fig. A.1, that is, for $423 \mathrm{~km} \mathrm{~s}^{-1}$ and $179 \mathrm{~km} \mathrm{~s}^{-1}$ as typical values of the Alfvén and sound speed at the $25 \mathrm{MHz}$ level in the corona, respectively. For this case, $M_{\mathrm{A}}^{*}>1.053$.

With the expression of the jump of the temperature across the shock (see Eq. (A.8)), the cross shock potential (see Eq. (15)) is found to be

$e \phi_{\mathrm{HT}}=k_{B} T_{1} \cdot \frac{\gamma}{2} \cdot \frac{v_{\mathrm{A} 1}^{2}}{c_{\mathrm{s} 1}^{2}} \cdot\left(M_{\mathrm{A}}^{*}\right)^{2} \cdot\left(1-\frac{1}{X^{2}}\right)$.

Then, one gets for the velocity $V_{\mathrm{e}}=\left(2 e \phi_{\mathrm{HT}} / m_{\mathrm{e}}\right)^{1 / 2}$

$V_{\mathrm{e}}=v_{\mathrm{th}, \mathrm{e}} \cdot \sqrt{\gamma \cdot \frac{v_{\mathrm{A} 1}^{2}}{c_{\mathrm{s} 1}^{2}} \cdot\left(M_{\mathrm{A}}^{*}\right)^{2} \cdot\left(1-\frac{1}{X^{2}}\right)}$. 